\title{
The denominators of Lagrangian surfaces in complex Euclidean plane
}

\author{
Katsuhiro Moriya* (moriya@math.tsukuba.ac.jp) \\ Institute of Mathematics, University of Tsukuba, 1-1-1 Tennodai, Tsukuba-shi, \\ Ibaraki-ken, 305-8571, Japan
}

\begin{abstract}
A quotient of two linearly independent quaternionic holomorphic sections of a quaternionic holomorphic line bundle over a Riemann surface is a conformal branched immersion from a Riemann surface to four-dimensional Euclidean space. On the assumption that a quaternionic holomorphic line bundle is associated with a Lagrangian branched immersion from a Riemann surface to complex Euclidean plane, we shall classify the denominators of Lagrangian branched immersion from a Riemann surface to complex Euclidean plane.
\end{abstract}

Keywords: Lagrangian surface, quaternionic holomorphic vector bundle, the CarlemanBers-Vekua system.

Mathematics Subject Classification (2000): Primary 53D12, Secondary 53C42.

\section{Introduction}

In this paper, we shall discuss a similarity between a Lagrangian branched immersion from a Riemann surface to complex Euclidean plane and a complex holomorphic function on a Riemann surface by the quaternionic theory of surfaces.

The quaternionic theory of surfaces in four-dimensional Euclidean space $\mathbb{R}^{4}$ is developed by Pedit and Pinkall [8], Burstall, Ferus, Leschke, Pedit, and Pinkall [1], and Ferus, Leschke, Pedit, and Pinkall [2]. This theory presents many new points of view on conformal geometry of surfaces in $\mathbb{R}^{4}$, where $\mathbb{R}^{4}$ is identified with the set $\mathbb{H}$ of quaternions.

In this theory, a right normal vector is defined for a conformal immersion from a Riemann surface $M$ to $\mathbb{H}$. A right normal vector is a quaternionic-valued function on $M$ whose square is -1 . It coincides with a part of the generalized Gauss map of the conformal immersion by taking a suitable decomposition of the Grassmanian manifold of twoplanes in $\mathbb{H}$ into a direct product of two spheres of dimension two. The tangent space of the immersion is preserved by the right multiplication of the right normal vector. Then a vector bundle endomorphism of the trivial (right) quaternionic line bundle $\underline{\mathbb{H}}$ over $M$ is defined by the right normal vector. This endomorphism is called a complex structure of $\underline{\mathbb{H}}$.

* Partly supported by the Grant-in-Aid for Young Scientists (B), The Ministry of Education, Culture, Sports, Science and Technology, Japan.

(C) 2007 Kluwer Academic Publishers. Printed in the Netherlands. 
For a smooth mapping from $M$ to $\mathbb{H}$, a right normal vector is defined uniquely on the set where the mapping is a conformal immersion. If the domain of a right normal vector can be extended to every point where the differential of the smooth mapping is not injective, then the smooth mapping is a conformal branched immersion by Lemma 1 in Section 2 .

A complex structure of $\underline{\mathbb{H}}$ plays a similar role to the complex structure of the space $\mathbb{C}$ of complex numbers. By a complex structure of

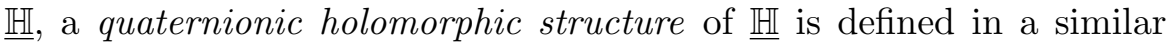
way to define the complex holomorphic structure of $\mathbb{C}$. A quaternionic holomorphic structure is a zero-order perturbation of a complex holomorphic structure of a complex Euclidean plane $\mathbb{C}^{2}$ identified with $\mathbb{H}$ (see p. 520 in [2]). This quaternionic holomorphic structure is called a Euclidean holomorphic structure in Peters [9]. A smooth section of $\underline{\mathbb{H}}$ in the kernel of a quaternionic holomorphic structure is called a quaternionic holomorphic section. When we consider a smooth section of $\underline{\mathbb{H}}$ as a smooth mapping from $M$ to $\mathbb{H}$, a non-constant quaternionic holomorphic section is a conformal branched immersion with a right normal vector. Hence a conformal branched immersion is a natural generalization of a complex holomorphic function on $M$. In the same way as a quotient of two complex holomorphic sections of a complex line trivial bundle is a complex holomorphic function except at the zeros of its denominator, a quotient of two linearly independent quaternionic holomorphic sections of $\underline{\mathbb{I}}$ with a complex structure is a conformal branched immersion with a right normal vector except at the zeros of its denominator by Example on p. 395 in [8].

We will look for a set of conformal branched immersions with a right normal vector satisfying a geometric property such that it is similar to a set of complex holomorphic function. Then it is expected that a set of Lagrangian branched immersion from $M$ to complex Euclidean plane $\mathbb{C}^{2}$ with a right normal vector is a candidate, where $\mathbb{C}^{2}$ is identified with $\mathbb{H}$. Indeed, we shall characterize a Lagrangian immersion by its right normal vector in Section 3. We define a complex structure by a right normal vector of a Lagrangian branched immersion. Then every quaternionic conjugate of non-constant quaternionic holomorphic section of $\underline{\mathbb{H}}$ is a Lagrangian branched immersion with the same right normal vector by the discussion in Section 2 .

We will consider the problem that whether the quotient of two Lagrangian branched immersions is a Lagrangian branched immersion. We should take a quotient of two linearly independent quaternionic holomorphic sections of $\underline{\mathbb{H}}$ with a complex structure defined by a right normal vector of a Lagrangian branched immersion. Then their quotient is not necessarily a Lagrangian branched immersion. Hence it is an interesting problem to classify the pairs of two quaternionic holomor- 
phic sections of $\underline{\mathbb{E}}$ such that their quotient is a Lagrangian branched immersion.

We shall devote this paper to classify quaternionic holomorphic sections of $\underline{\mathbb{I}}$ vanishing nowhere which are the denominators of Lagrangian branched immersions from $M$ to $\mathbb{C}^{2}$ with their right normal vector. This paper is organized as follows.

In Section 2, we shall review the quaternionic theory of conformal branched immersions from $M$ to $\mathbb{H}$ and rewrite Example on p. 395 in [8] to make it convenient for our use.

In Section 3, we shall characterize a Lagrangian immersion and a Hamiltonian-minimal Lagrangian immersion in terms of the quaternionic formulation. The notions of Hamiltonian-minimality is introduced by $\mathrm{Oh}[7]$.

In Section 4, we shall assume that a quaternionic holomorphic line bundle is associated with a Lagrangian branched immersion with a right normal vector. We shall classify the quaternionic holomorphic sections vanishing nowhere which are the denominators of Lagrangian branched immersions. In the case where $M$ is closed, the image of $M$ by a denominator is a torus (Theorem 1). In the case where $M$ is open, a complex-valued function is defined locally as a function of a complex holomorphic function on $M$ and Lagrangian angle mappings of a Lagrangian branched immersion and its denominator so that it is a solution to a differential equation called the Carleman-Bers-Vekua system in Rodin [10] (cf. Vekua [12]). A denominator is a mapping of this complex holomorphic function on $M$ and these Lagrangian angle mappings (Theorem 2).

In Section 5, we discuss the case where a Lagrangian branched immersion or its denominator is a Hamiltonian-minimal Lagrangian branched immersion. If both of them are Hamiltonian-minimal Lagrangian branched immersions, then the image of $M$ by a denominator is a plane or a torus (Theorem 3). If one is a Hamiltonian-minimal Lagrangian branched immersion and another is not a Hamiltonianminimal Lagrangian branched immersion, then we have a formula for the denominator as a mapping of a holomorphic function (Theorem 4 and Theorem 5).

In Section 6, we construct a numerator and obtain a Lagrangian branched immersion by Theorem 4 and Theorem 5 . 


\section{Quaternionic holomorphic line bundles}

We shall recall the quaternionic theory of surfaces by Pedit and Pinkall [8], Burstall, Ferus, Leschke, Pedit, and Pinkall [1], and Ferus, Leschke, Pedit, and Pinkall [2].

We denote by $\mathbb{R}$ the set of real numbers and by $\mathbb{H}$ the set of quaternions $\left\{a_{0}+a_{1} i+a_{2} j+a_{3} k \mid a_{0}, a_{1}, a_{2}, a_{3} \in \mathbb{R}\right\}$, where $i, j$, and $k$ are elements of $\mathbb{H}$ such that

$$
\begin{aligned}
& i^{2}=j^{2}=k^{2}=-1, \\
& i j=-j i=k, \quad j k=-k j=i, \quad k i=-i k=j .
\end{aligned}
$$

For a quaternion $a_{0}+a_{1} i+a_{2} j+a_{3} k$ such that $a_{0}, a_{1}, a_{2}$, and $a_{3} \in \mathbb{R}$, the quaternionic conjugate $\hat{a}$ of $a$, the real part $\operatorname{Re} a$ of $a$, and the imaginary part $\operatorname{Im} a$ of $a$ are defined by $\hat{a}=a_{0}-a_{1} i-a_{2} j-a_{3} k$, $\operatorname{Re} a=a_{0}$, and $\operatorname{Im} a=a_{1} i+a_{2} j+a_{3} k$ respectively. We denote by $\operatorname{Im} \mathbb{H}$ the set of imaginary parts of quaternions. The set of quaternions $\mathbb{H}$ is considered as the set of quadruplets of real numbers $\mathbb{R}^{4}$ by the identification of a quaternion $a_{0}+a_{1} i+a_{2} j+a_{3} k$ such that $a_{0}, a_{1}, a_{2}$, and $a_{3} \in \mathbb{R}$ with a quadruplet $\left(a_{0}, a_{1}, a_{2}, a_{3}\right)$ of real numbers. Let $q$ be a quaternionic sesquilinear product on $\mathbb{H}$ by $q(x, y)=\hat{x} y$ for every pair $(x, y)$ of quaternions. We define real-valued quadratic forms $\omega_{0}, \omega_{1}, \omega_{2}$, and $\omega_{3}$ by $q(x, y)=\omega_{0}(x, y)+\omega_{1}(x, y) i+\omega_{2}(x, y) j+\omega_{3}(x, y) k$. Then the quadratic form $\omega_{0}$ is the standard Euclidean inner product of $\mathbb{R}^{4}$. Let $\left(\mathbb{H}, \omega_{0}\right)$ be four-dimensional Euclidean space and $|a|=\left(\omega_{0}(a, a)\right)^{1 / 2}$ Euclidean norm of $a \in \mathbb{H}$.

The set $\left\{a_{0}+a_{1} i \mid a_{0}, a_{1} \in \mathbb{R}\right\}$ is considered as the set $\mathbb{C}$ of complex numbers. Then the set of quaternions $\mathbb{H}$ is considered as the set of pairs of complex numbers $\mathbb{C}^{2}$ by the identification of a quaternion $a_{0}+a_{1} i+$ $a_{2} j+a_{3} k$ such that $a_{0}, a_{1}, a_{2}$, and $a_{3} \in \mathbb{R}$ with a pair of complex numbers $\left(a_{0}+a_{1} i, a_{2}-a_{3} i\right)$. Then the quadratic $\omega_{1}$ is the standard symplectic form of $\mathbb{C}^{2}$ and $\omega_{0}+\omega_{1} i$ is the standard Hermitian inner product on $\mathbb{C}^{2}$.

Euclidean inner product $\omega_{0}$ induces the standard Riemannian metric of $\mathbb{R}^{4}$. We use the same notation $\omega_{0}$ for this Riemannian metric. Similarly, we use the same notation $\omega_{1}$ for the standard symplectic structure of $\mathbb{C}^{2}$ induced by the symplectic form $\omega_{1}$ on $\mathbb{C}^{2}$. Then $\omega_{0}+\omega_{1} i$ is the standard Hermitian metric of $\mathbb{C}^{2}$.

Let $(M, g)$ be a two-dimensional oriented connected Riemannian manifold $M$ with a Riemannian metric $g, T M$ its tangent bundle, and $T^{*} M$ its cotangent bundle. Then there exists a complex structure $J^{T M}$ of $(M, g)$ such that the ordered pair $\left(\mathbf{e}, J^{T M} \mathbf{e}\right)$ is a positive orthonormal basis of $T_{p} M$ for every point $p$ in $M$ and every unit vector $\mathbf{e}$ in the fiber $T_{p} M$ of $T M$ at $p$. 
For a smooth vector bundle $V$ over $M$, we denote by $\Gamma(V)$ the set of smooth sections of $V$ and $\Omega^{n}(V)$ the set of smooth differential $n$ forms on $M$ with coefficients in $V(n=0,1,2)$. We define a mapping $*: \Omega^{1}(V) \rightarrow \Omega^{1}(V)$ by $* \omega=\omega \circ J^{T M}$ for every $\omega \in \Omega^{1}(V)$.

Let $\underline{\mathbb{H}}$ be the trivial (right) quaternionic line bundle $\underline{\mathbb{H}}$ over $M$. A smooth mapping $\phi: M \rightarrow \mathbb{H}$ is considered as a smooth section $\underline{\phi}$ of $\underline{\mathbb{H}}$. Let $L$ be a pair $\left(\underline{\mathbb{H}}, J^{L}\right)$ with a quaternionic vector bundle endomorphism $J^{L}$ of $\underline{\mathbb{H}}$. The endomorphism $J^{L}$ is called a complex structure of $L$ in $[1]$.

Let $T^{*} M \otimes_{\mathbb{R}} \underline{\mathbb{H}}$ be the tensor bundle of $T^{*} M$ and $\underline{\mathbb{H}}$ over $\mathbb{R}$ and $\zeta \underline{\phi}$ an element of $T^{*} M \otimes_{\mathbb{R}} \underline{\mathbb{H}}$ such that $\zeta \in T^{*} M$ and $\phi \in \underline{\mathbb{H}}$. A quaternionicvalued one-form on $M$ is a section of $T^{*} M \otimes_{\mathbb{R}} \underline{\mathbb{H}}$. We define a vector bundle endomorphism $J$ of $T^{*} M \otimes_{\mathbb{R}} \underline{\mathbb{H}}$ by the equation $J \zeta \underline{\phi}=\zeta J^{L} \underline{\phi}$. A quaternionic vector bundle $\bar{K} \underline{\mathbb{H}}$ is defined by

$$
\bar{K} \underline{\mathbb{H}}=\left\{\omega \in T^{*} M \otimes_{\mathbb{R}} \underline{\mathbb{H}} \mid * \omega=-J \omega\right\} .
$$

We define a quaternionic homomorphism $D: \Gamma(\underline{\mathbb{H}}) \rightarrow \Gamma(\bar{K} \underline{\mathbb{H}})$ by

$$
D(\underline{\phi})=\frac{1}{2}\{(\mathrm{~d} \phi)+J *(\mathrm{~d} \phi)\} .
$$

for every smooth mapping $\phi$ from $M$ to $\mathbb{H}$. Following Peters [9], we call the quaternionic homomorphism $D$ the Euclidean quaternionic holomorphic structure of $L$ and the pair $L=\left(\mathbb{H}, J^{L}\right)$ with its Euclidean quaternionic holomorphic structure $D$ a Euclidean quaternionic holomorphic line bundle. A smooth section $\phi$ of $L$ is called a quaternionic holomorphic section of $L$ if $D(\phi)=0$. We see that a constant section is a quaternionic holomorphic section.

A smooth mapping $f:(M, g) \rightarrow\left(\mathbb{H}, \omega_{0}\right)$ is called a conformal immersion on $M$ if $f$ is an immersion and there exists a pair $\left(N^{f}, R^{f}\right)$ of smooth mappings from $M$ to $S^{2}(1) \subset \operatorname{Im} \mathbb{H}$ such that

$$
\begin{aligned}
& \left(N^{f}\right)^{2}=\left(R^{f}\right)^{2}=-1, \\
& *(\mathrm{~d} f)=N^{f}(\mathrm{~d} f)=(\mathrm{d} f)\left(-R^{f}\right) .
\end{aligned}
$$

The smooth mappings $N^{f}$ and $R^{f}$ defined by the equation (2.1) are called the left normal vector of $f$ and the right normal vector of $f$ respectively (Definition 2 in [1]).

A point $p \in M$ is called a branch point of a smooth mapping $f:(M, g) \rightarrow\left(\mathbb{H}, \omega_{0}\right)$ if the differential mapping $(\mathrm{d} f)_{p}$ of $f$ at $p$ is the zero mapping. A non-constant smooth mapping $f:(M, g) \rightarrow\left(\mathbb{H}, \omega_{0}\right)$ is called a conformal branched immersion if every point $p \in M$ such that $(\mathrm{d} f)_{p}$ is not injective is a branch point and $f$ is a conformal immersion on $M$ except branch points. 
A right normal vector is not defined by the equation (2.1) at a point $p \in M$ such that $(\mathrm{d} f)_{p}$ is not injective.

LEMMA 1. Let $f:(M, g) \rightarrow\left(\mathbb{H}, \omega_{0}\right)$ be a non-constant smooth mapping. If there exists a mapping $R^{f}: M \rightarrow S^{2}(1) \subset \operatorname{Im} \mathbb{H}$ such that

$$
\left(R^{f}\right)^{2}=-1, \quad *(\mathrm{~d} f)=(\mathrm{d} f)\left(-R^{f}\right),
$$

then $f$ is a conformal branched immersion.

Proof. It is indicated on p. 8 in [1] that if $*(\mathrm{~d} f)=(\mathrm{d} f)\left(-R^{f}\right)$, then $f$ is conformal at every point $p \in M$ such that $(\mathrm{d} f)_{p}$ is injective. Let $p \in M$ be a point such that $(\mathrm{d} f)_{p}$ is not injective and $\left(u_{1}, u_{2}\right)$ is an isothermal coordinate around $p$ such that $J^{T M}\left(\partial / \partial u_{1}\right)=\partial / \partial u_{2}$. If $*(\mathrm{~d} f)=(\mathrm{d} f)(-R)$, then

$$
\frac{\partial f}{\partial u_{2}}(p)=\frac{\partial f}{\partial u_{1}}(p)\left(-R^{f}(p)\right) .
$$

Since $(\mathrm{d} f)_{p}$ is not injective and $R^{f}$ is a mapping from $M$ to $S^{2}(1) \subset$ $\operatorname{Im} \mathbb{H}$, we have

$$
\frac{\partial f}{\partial u_{2}}(p)=\frac{\partial f}{\partial u_{1}}(p)=0 .
$$

Hence $(\mathrm{d} f)_{p}=0$.

We call the mapping $f$ with a smooth mapping $R^{f}: M \rightarrow S^{2}(1) \subset \operatorname{Im} \mathbb{H}$ such that $*(\mathrm{~d} f)=(\mathrm{d} f)\left(-R^{f}\right)$ on $M$ a conformal branched immersion with a right normal vector $R^{f}$.

Let $f:(M, g) \rightarrow\left(\mathbb{H}, \omega_{0}\right)$ be a conformal branched immersion with its right normal vector $R^{f}$. We define a complex structure $J^{f}$ of $\underline{\mathbb{H}}$ by $J^{f} \underline{1}=\underline{R^{f}}$. Let $D^{f}$ be the Euclidean quaternionic holomorphic structure of $\underline{L^{f}}=\left(\mathbb{H}, J^{f}\right)$ and $\hat{\phi}$ a smooth section of $L^{f}$. Since $D^{f}(\hat{\phi})=$ $\left\{(\mathrm{d} \hat{\phi})+R^{f} *(\mathrm{~d} \hat{\phi})\right\} / 2$, a section $\hat{\phi}$ of $L^{f}$ is a non-constant quaternionic holomorphic section if and only if $\phi$ is a conformal branched immersion with its right normal vector $R^{f}$. Hence the section $\underline{\hat{f}}$ is a non-constant quaternionic holomorphic section of $L^{f}$.

Let $L$ be a Euclidean quaternionic holomorphic line bundle over $M$ with its complex structure $J^{L}$ defined by $J^{L} \underline{1}=\underline{R}$ for a smooth mapping $R: M \rightarrow S^{2}(1) \subset \operatorname{Im} \mathbb{H}$. The following Lemma 2 is a variant of Example on p. 395 in [8].

LEMMA 2. We assume that $\underline{\hat{v}}$ is a non-zero quaternionic holomorphic section of $L$ and $\hat{\mu}$ is a smooth section vanishing nowhere of $L$. $A$ smooth mapping $\lambda:(M, g) \rightarrow\left(\mathbb{H}, \omega_{0}\right)$ defined by the equation $\underline{\hat{\nu}}=\underline{\hat{\mu}} \hat{\lambda}$ is 
a conformal branched immersion with its right normal vector $\mu R \mu^{-1}$ if and only if $\hat{\mu}$ is linearly independent of $\underline{\hat{v}}$ and it is a quaternionic holomorphic section of $L$.

Proof. Evaluating the both side of the equation $\underline{\hat{v}}=\underline{\hat{\mu}} \hat{\lambda}$ by the Euclidean quaternionic holomorphic structure $D$ of $L$, we have

$$
\underline{0}=D(\underline{\hat{\mu}}) \hat{\lambda}+\frac{1}{2} \hat{\mu}\left\{(\mathrm{d} \hat{\lambda})+\hat{\mu}^{-1} R \hat{\mu} *(\mathrm{~d} \hat{\lambda})\right\} .
$$

Hence Lemma 2 holds.

\section{Lagrangian surfaces}

We shall describe a conformal Lagrangian immersion from $(M, g)$ to $\left(\mathbb{C}^{2}, \omega_{0}\right)$ in terms of quaternions.

We identify $\mathbb{H}$ with $\mathbb{C}^{2}$ by the identification of a quaternion $a_{0}+$ $a_{1} i+a_{2} j+a_{3} k$ such that $a_{0}, a_{1}, a_{2}$, and $a_{3} \in \mathbb{R}$ with a pair of complex numbers $\left(a_{0}+a_{1} i, a_{2}-a_{3} i\right)$. A conformal immersion $f:(M, g) \rightarrow\left(\mathbb{H}, \omega_{0}\right)$ is called a Lagrangian immersion if

$$
\omega_{0}\left(\left\{(\mathrm{~d} f)_{p}(X)\right\} i,(\mathrm{~d} f)_{p}(Y)\right)=0,
$$

for every point $p \in M$ and every pair $(X, Y)$ of vectors $X$ and $Y \in$ $T_{p} M$. A conformal branched immersion $f:(M, g) \rightarrow\left(\mathbb{H}, \omega_{0}\right)$ is called a Lagrangian branched immersion if $f$ is a Lagrangian immersion on $M$ except at branch points.

We shall rephrase this definition in terms of quaternions. Let $\mathbb{Z}$ be the set of integers and $\mathbb{R} / 2 \pi \mathbb{Z}$ the quotient space of $\mathbb{R}$ by $2 \pi \mathbb{Z}=$ $\{2 \pi n \mid n \in \mathbb{Z}\}$. Let $f:(M, g) \rightarrow\left(\mathbb{H}, \omega_{0}\right)$ be a conformal immersion. We make another identification of $\mathbb{C}^{2}$ with $\mathbb{H}$ by the identification of $\left(z_{0}, z_{1}\right) \in \mathbb{C}^{2}$ with $\tau\left(z_{0}+j z_{1}\right) \tau^{-1}$, where $\tau=i+j$. Under this identification, Hélein and Romon [5] showed that a conformal immersion $\tilde{f}=\tau f \tau^{-1}$ is a Lagrangian immersion if and only if $(\mathrm{d} \tilde{f})=r(\mathrm{~d} z) e^{\theta j / 2}$ for a local complex holomorphic coordinate $z$ of $M$, a quaternionicvalued function $r$, and a smooth mapping $\theta: M \rightarrow \mathbb{R} / 2 \pi \mathbb{Z}$. The mapping $\theta$ is called the Lagrangian angle mapping of $f$. If the Lagrangian angle mapping is constant, then $f(M)$ is a Lagrangian plane. Let $h$ be the Riemannian metric of $\mathbb{R} / 2 \pi \mathbb{Z}$ induced by the standard Riemannian metric of $\mathbb{R}$. If the map $\theta:(M, g) \rightarrow(\mathbb{R} / 2 \pi \mathbb{Z}, h)$ is harmonic, then $f$ is called Hamiltonian-minimal Lagrangian immersion (see Hélein and Romon [6]). We see that

$$
*(\mathrm{~d} \tilde{f})=r(\mathrm{~d} z) i e^{\theta j / 2}=(\mathrm{d} \tilde{f}) e^{-\theta j / 2} i e^{\theta j / 2}=(\mathrm{d} \tilde{f}) i e^{\theta j} .
$$


Coming back to the identification of $\mathbb{C}^{2}$ with $\mathbb{H}$ by the identification $\left(z_{0}, z_{1}\right) \in \mathbb{C}^{2}$ with $z_{0}+j z_{1} \in \mathbb{H}$, we have

$$
*(\mathrm{~d} f)=(\mathrm{d} f) \tau^{-1} i e^{\theta j} \tau=(\mathrm{d} f) j e^{\theta i} .
$$

Hence the right normal vector of $f$ is $-j e^{\theta i}$. We define a mapping $\beta: M \rightarrow \mathbb{R} / 2 \pi \mathbb{Z}$ by $\beta=\theta+\pi$. Then the right normal vector of $f$ is $j e^{\beta i}$ and $f$ is Hamiltonian-minimal if and only if $\beta$ is harmonic.

\section{Lagrangian line bundles}

We shall classify the denominators of Lagrangian branched immersions from $(M, g)$ to $\left(\mathbb{H}, \omega_{0}\right)$.

Let $L$ be a Euclidean quaternionic holomorphic line bundle $L$ over a Riemann surface $M$ with complex structure $J^{L}$. We call $L$ a Lagrangian line bundle if $J^{L}$ is defined by $J^{L} \underline{1}=j e^{\beta i}$ with a smooth mapping $\beta: M \rightarrow \mathbb{R} / 2 \pi \mathbb{Z}$. A non-constant quaternionic holomorphic section of a Lagrangian line bundle with its complex structure defined by $J^{L} \underline{1}=$ $\underline{j e^{\beta i}}$ is identified with a Lagrangian branched immersion with a right normal vector $j e^{\beta i}$.

LEMMA 3. We assume that $\underline{\hat{\nu}}$ is a non-zero quaternionic holomorphic section of a Lagrangian line bundle $L$ with its complex structure $J^{L}$ defined by $J^{L} \underline{1}=j e^{\beta i}$ and that $\hat{\mu}$ is a nowhere-vanishing smooth section of $L$. A mapping $\overline{\lambda:(M}, g) \rightarrow\left(\bar{H}, \omega_{0}\right)$ defined by the equation $\hat{\nu}=\hat{\mu} \hat{\lambda}$ is a Lagrangian branched immersion with its right normal vector $j e^{\gamma i}$ if and only if $\underline{\hat{\mu}}$ is linearly independent of $\underline{\hat{v}}$ and

$$
\begin{aligned}
& \mu=\mu_{0} e^{(\beta-\gamma) i / 2}+j \mu_{1} e^{(\beta+\gamma) i / 2}, \\
& \mu_{0}(-*(\mathrm{~d} \beta)+*(\mathrm{~d} \gamma))=\mu_{1}((\mathrm{~d} \beta)+(\mathrm{d} \gamma)),
\end{aligned}
$$

where $\mu_{0}$ and $\mu_{1}$ are real-valued functions on $M$ such that $\mu_{0}-\mu_{1} i$ is a complex holomorphic function vanishing nowhere on $M$.

Proof. It is an immediate consequence of Lemma 2 that a mapping $\lambda$ is a Lagrangian branched immersion with its right normal vector $j e^{\gamma i}$ if and only if $\underline{\hat{\mu}}$ is linearly independent of $\underline{\hat{\nu}}, \underline{\hat{\mu}}$ is a nowhere-vanishing, quaternionic holomorphic section of $L$ satisfying $\mu j e^{\beta i} \mu^{-1}=j e^{\gamma i}$. We rewrite the last equation.

Let $F_{0}$ and $F_{1}$ be complex-valued functions on $M$ such that $\mu=F_{0}+$ $j F_{1}$. Then the equation $\mu j e^{\beta i} \mu^{-1}=j e^{\gamma i}$ is equivalent to the equation

$$
-\bar{F}_{1} e^{\beta i}+j \bar{F}_{0} e^{\beta i}=-F_{1} e^{-\gamma i}+j F_{0} e^{\gamma i} .
$$


Then the mapping $\mu: M \rightarrow \mathbb{H}$ given by the equation (4.1) is the solution to this equation.

A section $\hat{\mu}$ of $L$ defined by (4.1) is a quaternionic holomorphic section if and only if

$$
\begin{aligned}
& -e^{(\beta-\gamma) i / 2} *\left(\mathrm{~d} \mu_{1}\right)+\frac{1}{2} \mu_{1} i e^{(\beta-\gamma) i / 2}(*(\mathrm{~d} \beta)+*(\mathrm{~d} \gamma)) \\
& +j\left\{e^{(\beta+\gamma) i / 2} *\left(\mathrm{~d} \mu_{0}\right)-\frac{1}{2} \mu_{0} i e^{(\beta+\gamma) i / 2}(*(\mathrm{~d} \beta)-*(\mathrm{~d} \gamma))\right\} \\
& =e^{(\beta-\gamma) i / 2}\left(\mathrm{~d} \mu_{0}\right)+\frac{1}{2} \mu_{0} i e^{(\beta-\gamma) i / 2}((\mathrm{~d} \beta)-(\mathrm{d} \gamma)) \\
& +j\left\{e^{(\beta+\gamma) i / 2}\left(\mathrm{~d} \mu_{1}\right)+\frac{1}{2} \mu_{1} i e^{(\beta+\gamma) i / 2}((\mathrm{~d} \beta)+(\mathrm{d} \gamma))\right\} .
\end{aligned}
$$

This equation is equivalent to the system of equations (4.2) and

$$
*\left(\mathrm{~d} \mu_{0}\right)=\left(\mathrm{d} \mu_{1}\right) .
$$

Since this equation is equivalent to the equation

$$
\left(\mathrm{d}\left(\mu_{0}-\mu_{1} i\right)\right)+i *\left(\mathrm{~d}\left(\mu_{0}-\mu_{1} i\right)\right)=0,
$$

$\mu_{0}-\mu_{1} i$ is a complex holomorphic function. Since the section $\hat{\mu}$ vanishes nowhere on $M$ by the assumption, the function $\mu_{0}-\mu_{1} i$ vanishes nowhere on $M$.

We shall classify the branch points of a smooth mapping $\mu$ defined by (4.1) and (4.2) with real-valued functions $\mu_{0}$ and $\mu_{1}$ on $M$ such that $\mu_{0}-\mu_{1} i$ is a nowhere vanishing complex holomorphic function. Since

$$
\begin{aligned}
(\mathrm{d} \mu) & =\left(\mathrm{d} \mu_{0}\right) e^{(\beta-\gamma) i / 2}+\frac{1}{2} \mu_{0} i e^{(\beta-\gamma) i / 2}((\mathrm{~d} \beta)-(\mathrm{d} \gamma)) \\
& +j\left\{\left(\mathrm{~d} \mu_{1}\right) e^{(\beta+\gamma) i / 2}+\frac{1}{2} \mu_{1} i e^{(\beta+\gamma) i / 2}((\mathrm{~d} \beta)+(\mathrm{d} \gamma))\right\}
\end{aligned}
$$

a point $p \in M$ is a branch point of $\mu$ if and only if

$$
\begin{aligned}
& \left(\mathrm{d} \mu_{0}\right)_{p}=0, \quad\left(\mathrm{~d} \mu_{1}\right)_{p}=0, \\
& \mu_{0}(p)\left((\mathrm{d} \beta)_{p}-(\mathrm{d} \gamma)_{p}\right)=0, \quad \mu_{1}(p)\left((\mathrm{d} \beta)_{p}+(\mathrm{d} \gamma)_{p}\right)=0 .
\end{aligned}
$$

Hence a point $p \in M$ is a branch point of $\mu$ if and only if a point $p$ is a branch point of $\mu_{0}-\mu_{1} i$ and

$$
\begin{aligned}
& \mu_{0}(p)=0 \text { and }(\mathrm{d} \beta)_{p}+(\mathrm{d} \gamma)_{p}=0 \\
& \mu_{1}(p)=0 \text { and }(\mathrm{d} \beta)_{p}-(\mathrm{d} \gamma)_{p}=0,
\end{aligned}
$$

or

$$
(\mathrm{d} \beta)_{p}=(\mathrm{d} \gamma)_{p}=0 .
$$


We shall classify the denominators of Lagrangian branched immersions with a right normal vector. Let $\underline{\hat{v}}$ be a non-zero quaternionic holomorphic section of a Lagrangian line bundle $L$ with its complex structure $J^{L}$ defined by $J^{L} \underline{1}=\underline{j e^{\beta i}}$ and $\underline{\hat{\mu}}$ a nowhere-vanishing smooth section of $L$.

THEOREM 1. We assume that $M$ is a closed Riemann surface. The mapping $\lambda:(M, g) \rightarrow\left(\mathbb{H}, \omega_{0}\right)$ defined by the equation $\hat{\nu}=\hat{\mu} \hat{\lambda}$ is a Lagrangian branched immersion with its right normal vector $j e^{\gamma i}$ if and only if $\hat{\mu}$ is linearly independent of $\underline{\hat{\nu}}$ and $\mu=\mu_{0} e^{(\beta-\gamma) i / 2}+j \mu_{1} e^{(\beta+\gamma) i / 2}$ with real constants $\mu_{0}$ and $\mu_{1}$ such that $\left(\mu_{0}\right)^{2}+\left(\mu_{1}\right)^{2} \neq 0$ and that $\Psi=\mu_{0}(\beta-\gamma)+\mu_{1}(\beta+\gamma) i$ is a complex holomorphic mapping from $M$ to the torus $\mathbb{C} / \Lambda$ with $\Lambda=\left\{2 \pi \mu_{0} n+2 \pi \mu_{1} m i \mid n, m \in \mathbb{Z}\right\}$.

Proof. By Lemma 3, the mapping $\lambda$ is a Lagrangian branched immersion with its right normal vector $j e^{\gamma i}$ if and only if $\hat{\mu}$ is linearly independent of $\underline{\hat{\nu}}$ and $\mu$ is defined by (4.1) and (4.2) with real-valued function $\mu_{0}$ and $\mu_{1}$ on $M$ such that $\mu_{0}-\mu_{1} i$ is a complex holomorphic function vanishing nowhere on $M$. Hence $\mu_{0}$ and $\mu_{1}$ are real constants. Since $\mu$ vanishes nowhere, $\left(\mu_{0}\right)^{2}+\left(\mu_{1}\right)^{2} \neq 0$. Then the mapping $\Psi=\mu_{0}(\beta-\gamma)+\mu_{1}(\beta+\gamma) i$ is a non-constant complex holomorphic mapping from $M$ to $\mathbb{C} / \Lambda$. Indeed, the equation (4.2) is equivalent to the equation

$$
*\left(\mathrm{~d}\left\{\mu_{0}(-\beta+\gamma)\right\}\right)=\left(\mathrm{d}\left\{\mu_{1}(\beta+\gamma)\right\}\right) .
$$

This is equivalent to $\Psi$ being a complex holomorphic mapping from $M$ to $\mathbb{C} / \Lambda$.

We see that the Lagrangian branched immersions $\mu, \nu$, and $\lambda$ in the above theorem are Hamiltonian-minimal and that $\mu(M)$ is a torus. If $\Psi$ is non-constant, then the total branching order of $\Psi$ is two times the genus of $M$ by the Riemann-Hurwitz formula on p.140 in [3].

Next, we discuss the case where $M$ is an open Riemann surface. Let $\bar{\partial}$ be a mapping from the set of smooth complex-valued functions on $M$ to the set of smooth complex-valued one-forms of $(0,1)$-type on $M$ defined by $\bar{\partial}=2^{-1}(\mathrm{~d}+i * \mathrm{~d})$. Then a differential equation

$$
\bar{\partial} \psi=\psi a+\bar{\psi} b,
$$

with complex-valued one-forms $a$ and $b$ of $(0,1)$-type for a complexvalued function $\psi$ on $M$ is called the Carleman-Bers-Vekua system and a solution $\psi$ to the equation is called a generalized analytic function in Rodin [12] (cf, Vekua [10]).

On a sufficiently small open set of $M$, we may consider the mapping $\Psi=\mu_{0}(\beta-\gamma)+\mu_{1}(\beta+\gamma) i$ as a complex-valued function. 
THEOREM 2. We assume that $M$ is an open Riemann surface. The mapping $\lambda:(M, g) \rightarrow\left(\mathbb{H}, \omega_{0}\right)$ defined by the equation $\hat{\nu}=\hat{\mu} \hat{\lambda}$ is a Lagrangian branched immersion with its right normal vector $j e^{\gamma i}$ if and only if $\hat{\hat{\mu}}$ is linearly independent of $\underline{\hat{v}}$ and $\mu=\mu_{0} e^{(\beta-\gamma) i / 2}+j \mu_{1} e^{(\beta+\gamma) i / 2}$ with real-valued functions $\mu_{0}$ and $\mu_{1}$ on $M$ such that

- the function $\mu_{0}-\mu_{1} i$ is a complex holomorphic function on $M$ vanishing nowhere,

- the equation $(\mathrm{d} \beta)+(\mathrm{d} \gamma)=0$ holds on $\left\{p \in M \mid \mu_{0}(p)=0\right\}$,

- the equation $(\mathrm{d} \beta)-(\mathrm{d} \gamma)=0$ holds on $\left\{p \in M \mid \mu_{1}(p)=0\right\}$,

and that

- a mapping $\Psi=\mu_{0}(\beta-\gamma)+\mu_{1}(\beta+\gamma) i$ is a generalized analytic function for the Carleman-Bers-Vekua system

$$
\bar{\partial} \Psi=\Psi \frac{\bar{\partial} \log \left(\mu_{0} \mu_{1}\right)}{2}+\bar{\Psi} \frac{\bar{\partial} \log \left(\mu_{0} \mu_{1}^{-1}\right)}{2},
$$

on every sufficiently small open set of $\left\{p \in M \mid \mu_{0}(p) \mu_{1}(p) \neq 0\right\}$.

Proof. On the set $\left\{p \in M \mid \mu_{0}(p)=0\right\}$, the equation (4.2) is equivalent to the equation $\mu_{1}((\mathrm{~d} \beta)+(\mathrm{d} \gamma))=0$. Since $\left(\mu_{0}\right)^{2}+\left(\mu_{1}\right)^{2} \neq 0$, the equation (4.2) is equivalent to the equation $(\mathrm{d} \beta)+(\mathrm{d} \gamma)=0$. Similarly, the equation (4.2) is equivalent to $(\mathrm{d} \beta)-(\mathrm{d} \gamma)=0$ on $\left\{p \in M \mid \mu_{1}(p)=\right.$ $0\}$.

On a sufficiently small open set of a point $p$ with $\mu_{0}(p) \mu_{1}(p) \neq 0$, we define local real-valued functions $\eta$ and $\xi$ by $\eta=\beta-\gamma$ and $\xi=\beta+\gamma$. Then $\Psi=\mu_{0} \eta+\mu_{1} \xi i_{1}$ and the equation (4.2) is equivalent to the equation $\mu_{0} *(\mathrm{~d} \eta)=-\mu_{1}(\mathrm{~d} \xi)$. Since

$$
\left(\mathrm{d}\left(\mu_{0} \eta\right)\right)=\eta\left(\mathrm{d} \mu_{0}\right)+\mu_{0}(\mathrm{~d} \eta), \quad\left(\mathrm{d}\left(\mu_{1} \xi\right)\right)=\xi\left(\mathrm{d} \mu_{1}\right)+\mu_{1}(\mathrm{~d} \xi),
$$

the equation (4.2) is equivalent to the equation

$$
*\left(\mathrm{~d}\left(\mu_{0} \eta\right)\right)-\mu_{0} \eta *\left(\mathrm{~d} \log \mu_{0}\right)=-\left(\mathrm{d}\left(\mu_{1} \xi\right)\right)+\mu_{1} \xi\left(\mathrm{d} \log \mu_{1}\right) .
$$

Then the equation (4.2) is equivalent to

$$
\begin{aligned}
2 \bar{\partial} \Psi= & (\mathrm{d} \Psi)+i *(\mathrm{~d} \Psi) \\
= & \left(\mathrm{d}\left(\mu_{0} \eta\right)\right)+i\left(\mathrm{~d}\left(\mu_{1} \xi\right)\right)+i *\left(\mathrm{~d}\left(\mu_{0} \eta\right)\right)-*\left(\mathrm{~d}\left(\mu_{1} \xi\right)\right) \\
= & \mu_{0} \eta\left(\mathrm{d} \log \mu_{0}\right)-\mu_{1} \xi *\left(\mathrm{~d} \log \mu_{1}\right) \\
& +i\left\{\mu_{0} \eta *\left(\mathrm{~d} \log \mu_{0}\right)+\mu_{1} \xi\left(\mathrm{d} \log \mu_{1}\right)\right\} \\
= & \mu_{0} \eta\left(\left(\mathrm{d} \log \mu_{0}\right)+i *\left(\mathrm{~d} \log \mu_{0}\right)\right)
\end{aligned}
$$




$$
\begin{aligned}
& +\mu_{1} \xi\left(-*\left(\mathrm{~d} \log \mu_{1}\right)+i\left(\mathrm{~d} \log \mu_{1}\right)\right) \\
= & (\Psi+\bar{\Psi})\left(\bar{\partial} \log \mu_{0}\right)-(\Psi-\bar{\Psi})\left(-\bar{\partial} \log \mu_{1}\right) \\
= & \Psi\left(\bar{\partial} \log \mu_{0}+\bar{\partial} \log \mu_{1}\right)+\bar{\Psi}\left(\bar{\partial} \log \mu_{0}-\bar{\partial} \log \mu_{1}\right) \\
= & \Psi\left\{\bar{\partial} \log \left(\mu_{0} \mu_{1}\right)\right\}+\bar{\Psi}\left\{\bar{\partial} \log \left(\mu_{0} \mu_{1}^{-1}\right)\right\} .
\end{aligned}
$$

Then Theorem 2 follows from Lemma 3.

\section{Formulae for denominators}

We shall discuss the case where $\lambda$ or its denominator $\mu$ is a Hamiltonianminimal Lagrangian branched immersion with a right normal vector. Throughout this section, we assume that $M$ is an open Riemann surface. We call a Lagrangian line bundle $L$ with its Lagrangian angle $\beta$ a Hamiltonian-minimal Lagrangian line bundle if $\beta$ is a harmonic mapping.

We shall rewrite the equation (4.2) in another way. Let $\mu_{0}$ and $\mu_{1}$ be real-valued functions on $M$ such that $\mu_{0}-\mu_{1} i$ is a complex holomorphic function vanishing nowhere on $M$ and let $M^{\prime}$ be the set of branch points of $\mu_{0}-\mu_{1} i$. Then the functions $\mu_{0}$ and $\mu_{1}$ are constant if and only if $M^{\prime}=M$ and not constant if and only if every element of $M^{\prime}$ is an isolated point. We assume that $\mu_{0}$ and $\mu_{1}$ are not constant. Then $\left(\mu_{0}, \mu_{1}\right)$ is an isothermal coordinate on $M \backslash M^{\prime}$. We define real-valued functions $\beta_{\mu_{k}}, \gamma_{\mu_{k}}, \beta_{\mu_{k} \mu_{l}}$ and $\gamma_{\mu_{k} \mu_{l}}$ on $M \backslash M^{\prime}$ by the equations

$$
\begin{aligned}
(\mathrm{d} \beta) & =\beta_{\mu_{0}}\left(\mathrm{~d} \mu_{0}\right)+\beta_{\mu_{1}}\left(\mathrm{~d} \mu_{1}\right), \\
(\mathrm{d} \gamma) & =\gamma_{\mu_{0}}\left(\mathrm{~d} \mu_{0}\right)+\gamma_{\mu_{1}}\left(\mathrm{~d} \mu_{1}\right), \\
\left(\mathrm{d} \beta_{\mu_{k}}\right) & =\beta_{\mu_{k} \mu_{0}}\left(\mathrm{~d} \mu_{0}\right)+\beta_{\mu_{k} \mu_{l}}\left(\mathrm{~d} \mu_{1}\right), \\
\left(\mathrm{d} \gamma_{\mu_{k}}\right) & =\gamma_{\mu_{k} \mu_{0}}\left(\mathrm{~d} \mu_{0}\right)+\gamma_{\mu_{k} \mu_{1}}\left(\mathrm{~d} \mu_{1}\right), \quad(k, l=0,1) .
\end{aligned}
$$

Then $\beta_{\mu_{0} \mu_{1}}=\beta_{\mu_{1} \mu_{0}}$ and $\gamma_{\mu_{0} \mu_{1}}=\gamma_{\mu_{1} \mu_{0}}$. The equation (4.2) on $M \backslash M^{\prime}$ is equivalent to the equation

$$
\left(\begin{array}{cc}
\mu_{1} & \mu_{0} \\
\mu_{0} & -\mu_{1}
\end{array}\right)\left(\begin{array}{c}
\gamma_{\mu_{0}} \\
\gamma_{\mu_{1}}
\end{array}\right)=\left(\begin{array}{cc}
-\mu_{1} & \mu_{0} \\
\mu_{0} & \mu_{1}
\end{array}\right)\left(\begin{array}{c}
\beta_{\mu_{0}} \\
\beta_{\mu_{1}}
\end{array}\right),
$$

on $M \backslash M^{\prime}$.

LEMMA 4. If the equation (5.1) holds on $M \backslash M^{\prime}$, then the system of equations

$$
\begin{aligned}
& 2 \gamma_{\mu_{1}}+\mu_{1}\left(\gamma_{\mu_{0} \mu_{0}}+\gamma_{\mu_{1} \mu_{1}}\right)=-\mu_{1}\left(\beta_{\mu_{0} \mu_{0}}+\beta_{\mu_{1} \mu_{1}}\right), \\
& 2 \gamma_{\mu_{0}}+\mu_{0}\left(\gamma_{\mu_{0} \mu_{0}}+\gamma_{\mu_{1} \mu_{1}}\right)=\mu_{0}\left(\beta_{\mu_{0} \mu_{0}}+\beta_{\mu_{1} \mu_{1}}\right),
\end{aligned}
$$

holds on $M \backslash M^{\prime}$. 
Proof. By the differentiation of the both side of the equation (5.1), we have a system of equations

$$
\begin{aligned}
& \left(\begin{array}{ll}
0 & 1 \\
1 & 0
\end{array}\right)\left(\begin{array}{l}
\gamma_{\mu_{0}} \\
\gamma_{\mu_{1}}
\end{array}\right)+\left(\begin{array}{cc}
\mu_{1} & \mu_{0} \\
\mu_{0} & -\mu_{1}
\end{array}\right)\left(\begin{array}{l}
\gamma_{\mu_{0} \mu_{0}} \\
\gamma_{\mu_{1} \mu_{0}}
\end{array}\right) \\
= & \left(\begin{array}{ll}
0 & 1 \\
1 & 0
\end{array}\right)\left(\begin{array}{l}
\beta_{\mu_{0}} \\
\beta_{\mu_{1}}
\end{array}\right)+\left(\begin{array}{cc}
-\mu_{1} & \mu_{0} \\
\mu_{0} & \mu_{1}
\end{array}\right)\left(\begin{array}{l}
\beta_{\mu_{0} \mu_{0}} \\
\beta_{\mu_{1} \mu_{0}}
\end{array}\right), \\
& \left(\begin{array}{cc}
1 & 0 \\
0 & -1
\end{array}\right)\left(\begin{array}{l}
\gamma_{\mu_{0}} \\
\gamma_{\mu_{1}}
\end{array}\right)+\left(\begin{array}{cc}
\mu_{1} & \mu_{0} \\
\mu_{0} & -\mu_{1}
\end{array}\right)\left(\begin{array}{l}
\gamma_{\mu_{0} \mu_{1}} \\
\gamma_{\mu_{1} \mu_{1}}
\end{array}\right) \\
= & \left(\begin{array}{cc}
-1 & 0 \\
0 & 1
\end{array}\right)\left(\begin{array}{l}
\beta_{\mu_{0}} \\
\beta_{\mu_{1}}
\end{array}\right)+\left(\begin{array}{cc}
-\mu_{1} & \mu_{0} \\
\mu_{0} & \mu_{1}
\end{array}\right)\left(\begin{array}{l}
\beta_{\mu_{0} \mu_{1}} \\
\beta_{\mu_{1} \mu_{1}}
\end{array}\right) .
\end{aligned}
$$

This system of equations is equivalent to the system of equations

$$
\begin{aligned}
\gamma_{\mu_{1}}+\mu_{1} \gamma_{\mu_{0} \mu_{0}}+\mu_{0} \gamma_{\mu_{1} \mu_{0}} & =\beta_{\mu_{1}}-\mu_{1} \beta_{\mu_{0} \mu_{0}}+\mu_{0} \beta_{\mu_{1} \mu_{0}}, \\
\gamma_{\mu_{0}}+\mu_{0} \gamma_{\mu_{0} \mu_{0}}-\mu_{1} \gamma_{\mu_{1} \mu_{0}} & =\beta_{\mu_{0}}+\mu_{0} \beta_{\mu_{0} \mu_{0}}+\mu_{1} \beta_{\mu_{1} \mu_{0}}, \\
\gamma_{\mu_{0}}+\mu_{1} \gamma_{\mu_{0} \mu_{1}}+\mu_{0} \gamma_{\mu_{1} \mu_{1}} & =-\beta_{\mu_{0}}-\mu_{1} \beta_{\mu_{0} \mu_{1}}+\mu_{0} \beta_{\mu_{1} \mu_{1}}, \\
-\gamma_{\mu_{1}}+\mu_{0} \gamma_{\mu_{0} \mu_{1}}-\mu_{1} \gamma_{\mu_{1} \mu_{1}} & =\beta_{\mu_{1}}+\mu_{0} \beta_{\mu_{0} \mu_{1}}+\mu_{1} \beta_{\mu_{1} \mu_{1}} .
\end{aligned}
$$

Lemma 4 follows from this system of equations.

We shall discuss the case where $\mu, \nu$ and $\lambda$ are Hamiltonian-minimal Lagrangian branched immersions. Let $\hat{\underline{\nu}}$ be a non-zero quaternionic holomorphic section of a Hamiltonian-minimal Lagrangian line bundle $L$ with its complex structure $J^{L}$ defined by $J^{L} \underline{1}=j e^{\beta i}$ and $\hat{\mu}$ a nowhere-vanishing quaternionic holomorphic sections of $\bar{L}$.

THEOREM 3. The mapping $\lambda:(M, g) \rightarrow\left(\mathbb{H}, \omega_{0}\right)$ defined by the equation $\hat{\nu}=\hat{\mu} \hat{\lambda}$ is a Hamiltonian-minimal Lagrangian branched immersion with its right normal vector $j e^{\gamma i}$ if and only if $\hat{\mu}$ is linearly independent of $\underline{\hat{\nu}}$ and $\mu=\mu_{0} e^{(\beta-\gamma) i / 2}+j \mu_{1} e^{(\beta+\gamma) i / 2}$ with real-valued functions $\mu_{0}$ and $\mu_{1}$ on $M$ such that

- the functions $\mu_{0}$ and $\mu_{1}$ are constants with $\left(\mu_{0}\right)^{2}+\left(\mu_{1}\right)^{2} \neq 0$ and $\Psi=\mu_{0}(\beta-\gamma)+\mu_{1}(\beta+\gamma) i$ is a complex holomorphic mapping from $M$ to $\mathbb{C} / \Lambda$ with $\Lambda=\left\{2 \pi \mu_{0} n+2 \pi \mu_{1} m i \mid n, m \in \mathbb{Z}\right\}$,

or

- the function $\mu_{0}-\mu_{1} i$ is a non-constant complex holomorphic function vanishing nowhere and $\beta$ and $\gamma$ are constant mappings.

Proof. Let $\lambda$ be a Hamiltonian-minimal Lagrangian branched immersion. If $\mu_{0}$ and $\mu_{1}$ are constants, then $\Psi$ is a complex holomorphic 
mapping from $M$ to $\mathbb{C} / \Lambda$ by (4.2) in the same way as the proof of Theorem 1 . We assume that $\mu_{0}-\mu_{1} i$ is a non-constant complex holomorphic function. By Lemma 4, we have

$$
2 \gamma_{\mu_{1}}=0, \quad 2 \gamma_{\mu_{0}}=0,
$$

on $M \backslash M^{\prime}$ since $\beta$ and $\gamma$ are harmonic mappings. Hence $\gamma$ is a constant mapping on $M \backslash M^{\prime}$. Then $-\mu_{0} *(\mathrm{~d} \beta)=\mu_{1}(\mathrm{~d} \beta)$ by the equation (4.2) on $M \backslash M^{\prime}$. Since $\mu_{0}(\mathrm{~d} \beta)=\mu_{1} *(\mathrm{~d} \beta)$, we have $\left\{\left(\mu_{0}\right)^{2}+\left(\mu_{1}\right)^{2}\right\}(\mathrm{d} \beta)=0$ on $M \backslash M^{\prime}$. Hence $(\mathrm{d} \beta)=0$ and $\beta$ is a constant mapping on $M \backslash M^{\prime}$. Since every element of $M^{\prime}$ is an isolated point and $\beta$ and $\gamma$ are smooth on $M$, both $\beta$ and $\gamma$ are constant mappings on $M$.

It is easy to see that the converse holds,

We shall discuss the case where $\mu$ and $\nu$ are Hamiltonian-minimal Lagrangian branched immersion and $\lambda$ is a Lagrangian branched immersion with its right normal vector $j e^{i \gamma}$ which is not Hamiltonianminimal. Let $\underline{\hat{\nu}}$ be a non-zero quaternionic holomorphic section of a Hamiltonian-minimal Lagrangian line bundle $L$ with its complex structure $J^{L}$ defined by $J^{L} \underline{1}=j e^{\beta i}$ and $\underline{\hat{\mu}}$ a nowhere-vanishing quaternionic holomorphic sections of $L$.

THEOREM 4. The mapping $\lambda:(M, g) \rightarrow\left(\mathbb{H}, \omega_{0}\right)$ defined by the equation $\hat{\nu}=\hat{\mu} \hat{\lambda}$ is a Lagrangian branched immersion with its right normal vector $j e^{\gamma i}$ which is not Hamiltonian-minimal if and only if $\hat{\mu}$ is linearly independent of $\underline{\hat{\nu}}$ and $\mu=\mu_{0} e^{(\beta-\gamma) i / 2}+j \mu_{1} e^{(\beta+\gamma) i / 2}$ with real-valued functions $\mu_{0}$ and $\mu_{1}$ on $M$ such that $\mu_{0}-\mu_{1} i$ is a non-constant complex holomorphic function vanishing nowhere on $M$ and mappings $\beta$ and $\gamma$ are given by the equations

$$
\begin{aligned}
& \beta\left(\mu_{0}, \mu_{1}\right)=A \frac{\left(\mu_{0}^{2}-\mu_{1}^{2}\right)}{\left(\mu_{0}^{2}+\mu_{1}^{2}\right)^{2}}+B, \\
& \gamma\left(\mu_{0}, \mu_{1}\right)=\frac{A}{\mu_{0}^{2}+\mu_{1}^{2}}+C,
\end{aligned}
$$

on $M$ for an arbitrary non-zero real number $A$ and arbitrary real numbers $B$ and $C$.

Proof. We assume that $\lambda$ is a Lagrangian branched immersion with its right normal vector $j e^{\gamma i}$ which is not a Hamiltonian-minimal Lagrangian branched immersion. If $\mu_{0}$ and $\mu_{1}$ are constant functions, then $\Psi=\mu_{0}(\beta-\gamma)+\mu_{1}(\beta+\gamma) i$ is a complex holomorphic mapping in the same way as the proof of Theorem 1. Then $\beta$ and $\gamma$ are harmonic mappings. Since $\gamma$ is not a harmonic mapping by the assumption, the functions $\mu_{0}$ and $\mu_{1}$ are not constant functions. 
Since $\beta$ is a harmonic mapping, we have

$$
\begin{aligned}
& 2 \gamma_{\mu_{1}}+\mu_{1}\left(\gamma_{\mu_{0} \mu_{0}}+\gamma_{\mu_{1} \mu_{1}}\right)=0, \\
& 2 \gamma_{\mu_{0}}+\mu_{0}\left(\gamma_{\mu_{0} \mu_{0}}+\gamma_{\mu_{1} \mu_{1}}\right)=0,
\end{aligned}
$$

on $M \backslash M^{\prime}$ by Lemma 4 . Then $\mu_{1} \gamma_{\mu_{0}}-\mu_{0} \gamma_{\mu_{1}}=0$. Hence $\gamma\left(\mu_{0}, \mu_{1}\right)=$ $\phi\left(\mu_{0}^{2}+\mu_{1}^{2}\right)$ on $M \backslash M^{\prime}$ for a smooth real-valued function $\phi$ on $\mathbb{R} \backslash\left\{\mu_{0}^{2}(p)+\right.$ $\left.\mu_{1}^{2}(p) \mid p \in M \backslash M^{\prime}\right\}$.

Since

$$
\begin{aligned}
& \gamma_{\mu_{0} \mu_{0}}\left(\mu_{0}, \mu_{1}\right)=4 \mu_{0}^{2} \phi^{\prime \prime}\left(\mu_{0}^{2}+\mu_{1}^{2}\right)+2 \phi^{\prime}\left(\mu_{0}^{2}+\mu_{1}^{2}\right), \\
& \gamma_{\mu_{1} \mu_{1}}\left(\mu_{0}, \mu_{1}\right)=4 \mu_{1}^{2} \phi^{\prime \prime}\left(\mu_{0}^{2}+\mu_{1}^{2}\right)+2 \phi^{\prime}\left(\mu_{0}^{2}+\mu_{1}^{2}\right),
\end{aligned}
$$

the equations (5.6) and (5.7) is equivalent to the equation

$$
t \phi^{\prime \prime}(t)+2 \phi^{\prime}(t)=0, \quad t=\mu_{0}^{2}+\mu_{1}^{2} .
$$

The solution to this equation is $\phi^{\prime}(t)=-A t^{-2}$ for a real number $A$. Since $\gamma$ is not a harmonic mapping, it is not a constant mapping. Then we obtain the equation (5.5) with a non-zero real number $A$ and a real number $C$ on $M \backslash M^{\prime}$. Since every element of $M^{\prime}$ is an isolated point and $\gamma$ is smooth on $M$, the equation (5.5) holds on $M$.

Since

$$
\begin{aligned}
& \gamma_{\mu_{0}}\left(\mu_{0}, \mu_{1}\right)=-\frac{2 A \mu_{0}}{\left(\mu_{0}^{2}+\mu_{1}^{2}\right)^{2}}, \\
& \gamma_{\mu_{1}}\left(\mu_{0}, \mu_{1}\right)=-\frac{2 A \mu_{1}}{\left(\mu_{0}^{2}+\mu_{1}^{2}\right)^{2}},
\end{aligned}
$$

we have

$$
\begin{aligned}
\left(\begin{array}{c}
\beta_{\mu_{0}} \\
\beta_{\mu_{1}}
\end{array}\right) & =\left(\begin{array}{cc}
-\mu_{1} & \mu_{0} \\
\mu_{0} & \mu_{1}
\end{array}\right)^{-1}\left(\begin{array}{cc}
\mu_{1} & \mu_{0} \\
\mu_{0} & -\mu_{1}
\end{array}\right)\left(\begin{array}{l}
-2 A \mu_{0}\left(\mu_{0}^{2}+\mu_{1}^{2}\right)^{-2} \\
-2 A \mu_{1}\left(\mu_{0}^{2}+\mu_{1}^{2}\right)^{-2}
\end{array}\right) \\
& =\frac{-2 A}{\left(\mu_{0}^{2}+\mu_{1}^{2}\right)^{3}}\left(\begin{array}{c}
\mu_{0}^{3}-3 \mu_{0} \mu_{1}^{2} \\
3 \mu_{0}^{2} \mu_{1}-\mu_{1}^{3}
\end{array}\right),
\end{aligned}
$$

on $M \backslash M^{\prime}$ by the equation (5.1). Since

$$
\begin{aligned}
& \frac{\mu_{0}^{3}-3 \mu_{0} \mu_{1}^{2}}{\left(\mu_{0}^{2}+\mu_{1}^{2}\right)^{3}}=\frac{\mu_{0}\left(\mu_{0}^{2}+\mu_{1}^{2}-4 \mu_{1}^{2}\right)}{\left(\mu_{0}^{2}+\mu_{1}^{2}\right)^{3}}=\frac{\mu_{0}}{\left(\mu_{0}^{2}+\mu_{1}^{2}\right)^{2}}+\frac{\mu_{0}\left(-4 \mu_{1}^{2}\right)}{\left(\mu_{0}^{2}+\mu_{1}^{2}\right)^{3}} \\
& =\frac{\partial}{\partial \mu_{0}} \frac{-1}{2\left(\mu_{0}^{2}+\mu_{1}^{2}\right)}+\frac{\partial}{\partial \mu_{0}} \frac{\mu_{1}^{2}}{\left(\mu_{0}^{2}+\mu_{1}^{2}\right)^{2}},
\end{aligned}
$$

we have

$$
\begin{aligned}
& \beta\left(\mu_{0}, \mu_{1}\right)=-2 A\left(\frac{-1}{2\left(\mu_{0}^{2}+\mu_{1}^{2}\right)}+\frac{\mu_{1}^{2}}{\left(\mu_{0}^{2}+\mu_{1}^{2}\right)^{2}}\right)+E\left(\mu_{1}\right) \\
& =-2 A\left(\frac{1}{2\left(\mu_{0}^{2}+\mu_{1}^{2}\right)}-\frac{\mu_{0}^{2}}{\left(\mu_{0}^{2}+\mu_{1}^{2}\right)^{2}}\right)+E\left(\mu_{1}\right),
\end{aligned}
$$


where $E\left(\mu_{1}\right)$ is a differentiable function of $\mu_{1}$. Then

$$
\begin{aligned}
& \beta_{\mu_{1}}\left(\mu_{0}, \mu_{1}\right)=-2 A\left(\frac{-\mu_{1}}{\left(\mu_{0}^{2}+\mu_{1}^{2}\right)^{2}}+\frac{4 \mu_{0}^{2} \mu_{1}}{\left(\mu_{0}^{2}+\mu_{1}^{2}\right)^{3}}\right)+\frac{\partial}{\partial \mu_{1}} E\left(\mu_{1}\right) \\
& =\frac{-2 A\left(3 \mu_{0}^{2} \mu_{1}-\mu_{1}^{3}\right)}{\left(\mu_{0}^{2}+\mu_{1}^{2}\right)^{3}}+\frac{\partial}{\partial \mu_{1}} E\left(\mu_{1}\right) .
\end{aligned}
$$

Hence $E\left(\mu_{1}\right)$ is a constant and the equation (5.4) is satisfied on $M \backslash M^{\prime}$ for a non-zero real number $A$ and a real number $B$. Since every element of $M^{\prime}$ is an isolated point and $\beta$ is smooth, the equation (5.4) holds on $M$.

Conversely, we assume that $\beta$ and $\gamma$ satisfies the equations (5.4) and (5.5). Then we see that $\beta$ is a harmonic mapping and that the equation (4.2) holds by a direct calculation.

We discuss the case where $\mu$ and $\nu$ are Lagrangian branched immersions which are not Hamiltonian-minimal and $\lambda$ is a Hamiltonianminimal Lagrangian branched immersion. Let $\underline{\hat{\nu}}$ be a non-zero quaternionic holomorphic section of a Lagrangian line bundle $L$ with its complex structure $J^{L}$ defined by $J^{L} \underline{1}=j e^{\beta i}$ which is not Hamiltonianminimal and $\underline{\hat{\mu}}$ a nowhere-vanishing quaternionic holomorphic sections of $L$.

THEOREM 5. The mapping $\lambda:(M, g) \rightarrow\left(\mathbb{H}, \omega_{0}\right)$ defined by the equation $\hat{\nu}=\hat{\mu} \hat{\lambda}$ is a Hamiltonian-minimal Lagrangian branched immersion with its right normal vector $j e^{\gamma i}$ if and only if $\hat{\mu}$ is linearly independent of $\hat{\hat{\nu}}$ and $\mu=\mu_{0} e^{(\beta-\gamma) i / 2}+j \mu_{1} e^{(\beta+\gamma) i / 2}$ with real-valued functions $\mu_{0}$ and $\mu_{1}$ on $M$ such that $\mu_{0}-\mu_{1} i$ is non-constant complex holomorphic function vanishing nowhere and mappings $\beta$ and $\gamma$ are given by the equations

$$
\begin{aligned}
& \beta\left(\mu_{0}, \mu_{1}\right)=A\left(\mu_{0}^{2}+\mu_{1}^{2}\right)+B, \\
& \gamma\left(\mu_{0}, \mu_{1}\right)=A\left(\mu_{0}^{2}-\mu_{1}^{2}\right)+C,
\end{aligned}
$$

on $M$ with a non-zero real number $A$ and real numbers $B$ and $C$.

Proof. We assume that $\lambda$ is a Hamiltonian-minimal Lagrangian immersion with its right normal vector $j e^{\gamma i}$. Since $\beta$ is not a harmonic mapping, we see that the functions $\mu_{0}$ and $\mu_{1}$ are not constant functions in a similar way as the proof of Theorem 4 .

Since $\gamma$ is a harmonic mapping, we have the equations

$$
\begin{aligned}
& 2 \gamma_{\mu_{0}}=\mu_{0}\left(\beta_{\mu_{0} \mu_{0}}+\beta_{\mu_{1} \mu_{1}}\right), \\
& 2 \gamma_{\mu_{1}}=-\mu_{1}\left(\beta_{\mu_{0} \mu_{0}}+\beta_{\mu_{1} \mu_{1}}\right),
\end{aligned}
$$


on $M \backslash M^{\prime}$ by Lemma 4 . Then $\mu_{1} \gamma_{\mu_{0}}+\mu_{0} \gamma_{\mu_{1}}=0$. Hence $\gamma\left(\mu_{0}, \mu_{1}\right)=$ $\phi\left(\mu_{0}^{2}-\mu_{1}^{2}\right)$ for a smooth real-valued function $\phi$ on $\mathbb{R} \backslash\left\{\mu_{0}^{2}(p)-\mu_{1}^{2}(p) \mid p \in\right.$ $\left.M \backslash M^{\prime}\right\}$. Since

$$
\begin{aligned}
\gamma_{\mu_{0}}\left(\mu_{0}, \mu_{1}\right) & =2 \mu_{0} \phi^{\prime}\left(\mu_{0}^{2}-\mu_{1}^{2}\right), \\
\gamma_{\mu_{1}}\left(\mu_{0}, \mu_{1}\right) & =-2 \mu_{1} \phi^{\prime}\left(\mu_{0}^{2}-\mu_{1}^{2}\right), \\
\gamma_{\mu_{0} \mu_{0}}\left(\mu_{0}, \mu_{1}\right) & =4 \mu_{0}^{2} \phi^{\prime \prime}\left(\mu_{0}^{2}-\mu_{1}^{2}\right)+2 \phi^{\prime}\left(\mu_{0}^{2}-\mu_{1}^{2}\right), \\
\gamma_{\mu_{1} \mu_{1}}\left(\mu_{0}, \mu_{1}\right) & =4 \mu_{1}^{2} \phi^{\prime \prime}\left(\mu_{0}^{2}-\mu_{1}^{2}\right)-2 \phi^{\prime}\left(\mu_{0}^{2}-\mu_{1}^{2}\right),
\end{aligned}
$$

we have

$$
\gamma_{\mu_{0} \mu_{0}}\left(\mu_{0}, \mu_{1}\right)+\gamma_{\mu_{1} \mu_{1}}\left(\mu_{0}, \mu_{1}\right)=4\left(\mu_{0}^{2}+\mu_{1}^{2}\right) \phi^{\prime \prime}\left(\mu_{0}^{2}-\mu_{1}^{2}\right)=0 .
$$

Hence the equation (5.9) holds on $M \backslash M^{\prime}$ for a non-zero real number $A$ and a real number $C$. Since every element of $M^{\prime}$ is an isolated point and $\gamma$ is smooth on $M$, the equation (5.9) holds on $M$.

By the equation (5.1), we have the equation

$$
\left(\begin{array}{l}
\beta_{\mu_{0}} \\
\beta_{\mu_{1}}
\end{array}\right)=2 A\left(\begin{array}{l}
\mu_{0} \\
\mu_{1}
\end{array}\right) .
$$

Hence the equation (5.8) holds for a non-zero real number $A$ and a real number $B$ on $M \backslash M^{\prime}$. Since every element of $M^{\prime}$ is an isolated point and $\beta$ is smooth, the equation (5.8) holds on $M$.

Conversely, we assume that $\beta$ and $\gamma$ are given by the equations (5.8) and (5.9) respectively. Then we see that the equation (4.2) holds and $\gamma$ is a harmonic mapping by a direct calculation.

\section{Examples}

We apply Theorem 4 and Theorem 5 to obtain examples of Lagrangian branched immersions. We calculate left normal vectors of the examples to see that there are examples with both conformal Maslov forms (see [4]) and non-conformal Maslov forms.

Let $f: M \rightarrow \mathbb{C}^{2}$ be a Lagrangian immersion with its left normal vector $N$ and its right normal vector $j e^{\beta i}$. The map $\left(N, j e^{\beta i}\right): M \rightarrow$ $S^{2}(1) \times S^{1}(1)$ is a decomposition of the generalized Gauss map of $f$, where $S^{1}(1)$ is a circle in $\{j(u+v i) \mid u, v \in \mathbb{R}\}$ with radius one centered at origin. Let $\omega_{1}$ is the symplectic form of $\mathbb{C}^{2}$ and $H$ the mean curvature vector of $f$. The one-form $\varpi$ on $M$ defined by $\varpi(X)=\omega_{1}(X, H) / \pi$ is called the Maslov form of $f$. A Maslov form $\varpi$ is said to be conformal 
if the tension field of the left normal vector $N$ of $f$ vanishes, or equivalently $\mathrm{d} *(\mathrm{~d} N)=h N$ with a real-valued function $h$ on $M$. Locally, this equation is equivalent to the equation

$$
N_{x x}+N_{y y}=k N
$$

where $(x, y)$ is a local coordinate of $M$ such that $x+y i$ is a local complex holomorphic coordinate and $k$ is a local real-valued function on $M$ (see [11] for example).

We use the following coordinate transformation. Let $\mu_{0}$ and $\mu_{1}$ be real-functions on $M$ such that $\mu_{0}-\mu_{1} i$ is a complex holomorphic function on $M$. Then $\left(\mu_{0}, \mu_{1}\right)$ is a coordinate of $M$ and $\mu_{0}-\mu_{1} i$ is a complex holomorphic coordinate except branch points of $\mu_{0}-\mu_{1} i$. Let $x=\mu_{0}\left(\mu_{0}^{2}+\mu_{1}^{2}\right)^{-1}$ and $y=\mu_{1}\left(\mu_{0}^{2}+\mu_{1}^{2}\right)^{-1}$. Then $(x, y)$ is a coordinate on $M$ such that $x+y i$ is a complex holomorphic coordinate of $M$ except branch point and zeros of $\mu_{0}-\mu_{1} i$. We see that $x_{0}^{2}+x_{1}^{2}=\left(\mu_{0}^{2}+\mu_{1}^{2}\right)^{-1}$.

Example 1. Let $\mu=\mu_{0} e^{(\beta-\gamma) i / 2}+j \mu_{1} e^{(\beta+\gamma) i / 2}$ with real-valued functions $\mu_{0}$ and $\mu_{1}$ on $M$ such that $\mu_{0}-\mu_{1} i$ is a non-constant complex holomorphic function vanishing nowhere on $M$. We assume that the mappings $\beta$ and $\gamma$ are given by the equations (5.4) and (5.5) with nonzero real number $A$ and $B=C=0$. Then

$$
\mu\left(\mu_{0}, \mu_{1}\right)=\mu_{0} e^{-A \mu_{1}^{2}\left(\mu_{0}^{2}+\mu_{1}^{2}\right)^{-2} i}+j \mu_{1} e^{A \mu_{0}^{2}\left(\mu_{0}^{2}+\mu_{1}^{2}\right)^{-2} i}
$$

is a Hamiltonian-minimal Lagrangian branched immersion with its right normal vector $j e^{\beta i}$ by Theorem 4 .

Let $(x, y)$ be a coordinate of $M$ such that $x=\mu_{0}\left(\mu_{0}^{2}+\mu_{1}^{2}\right)^{-1}$ and $y=\mu_{1}\left(\mu_{0}^{2}+\mu_{1}^{2}\right)^{-1}$. Since

$$
\begin{aligned}
& \mu=\frac{x}{x^{2}+y^{2}} e^{-A y^{2} i}+j \frac{y}{x^{2}+y^{2}} e^{A x^{2} i} \\
& \mu_{x}=\frac{-x^{2}+y^{2}}{\left(x^{2}+y^{2}\right)^{2}} e^{-A y^{2} i}+j \frac{2 x y\left\{-1+A\left(x^{2}+y^{2}\right) i\right\}}{\left(x^{2}+y^{2}\right)^{2}} e^{A x^{2} i}, \\
& \mu_{y}=\frac{2 x y\left\{-1-A\left(x^{2}+y^{2}\right) i\right\}}{\left(x^{2}+y^{2}\right)^{2}} e^{-A y^{2} i}+j \frac{x^{2}-y^{2}}{\left(x^{2}+y^{2}\right)^{2}} e^{A x^{2} i},
\end{aligned}
$$

the left normal vector of $\mu$ is

$$
\mu_{y} \mu_{x}^{-1}=-j e^{-A\left(x^{2}-y^{2}\right) i} .
$$

By the equation (6.1), we see that the Maslov form of $\mu$ is conformal. The section 1 of a Hamiltonian-minimal Lagrangian line bundle $L$ with its complex structure $J^{L}$ defined by $J^{L} \underline{1}=j e^{\beta i}$ is a non-zero quaternionic holomorphic section. We define a smooth mapping $\lambda$ by $\underline{1}=\underline{\hat{\mu}} \hat{\lambda}$. 


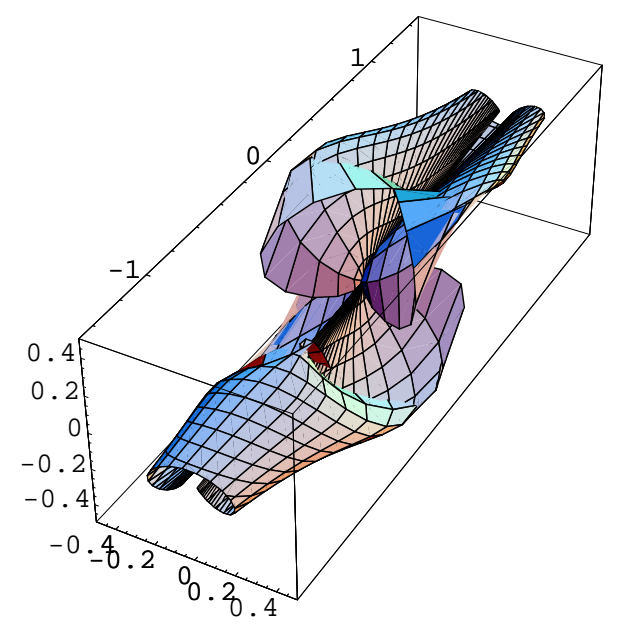

Figure 1. (Example 1) $U=\left\{x+y i \in \mathbb{C} \mid 0.5^{2} \leq x^{2}+y^{2} \leq 4^{2}\right\}, \mu_{0}=x, \mu_{1}=-y$, $A=1, \operatorname{Im} \mu: U \rightarrow \operatorname{Im} \mathbb{H}$.

Then

$$
\begin{aligned}
\lambda\left(\mu_{0}, \mu_{1}\right) & =\frac{1}{\mu_{0}^{2}+\mu_{1}^{2}} \\
& \times\left(\mu_{0} e^{A \mu_{1}^{2}\left(\mu_{0}^{2}+\mu_{1}^{2}\right)^{-2} i}-j \mu_{1} e^{A \mu_{0}^{2}\left(\mu_{0}^{2}+\mu_{1}^{2}\right)^{-2} i}\right)
\end{aligned}
$$

is a Lagrangian branched immersion with its right normal vector $j e^{\gamma i}$ which is not Hamiltonian-minimal by Theorem 4 .

Let $x=\mu_{0}\left(\mu_{0}^{2}+\mu_{1}^{2}\right)^{-1}$ and $y=\mu_{1}\left(\mu_{0}^{2}+\mu_{1}^{2}\right)^{-1}$. Then $(x, y)$ is a coordinate of $M$ such that $x+y i$ is a complex holomorphic coordinate except branch point of $\mu_{0}-\mu_{1} i$. Since

$$
\begin{aligned}
& \lambda=x e^{A y^{2} i}-j y e^{A x^{2}}, \\
& \lambda_{x}=e^{A y^{2} i}-j 2 A x y i e^{A x^{2} i}, \quad \lambda_{y}=2 A x y i e^{A y^{2} i}-j e^{A x^{2} i},
\end{aligned}
$$

the left normal vector of $\lambda$ is

$$
\lambda_{y} \lambda_{x}^{-1}=\frac{4 A x y i}{1+4 A^{2} x^{2} y^{2}}+j \frac{\left(4 A^{2} x^{2} y^{2}-1\right) e^{A\left(x^{2}-y^{2}\right) i}}{1+4 A^{2} x^{2} y^{2}} .
$$

After long computation, we see that the Maslov form of $\lambda$ is not conformal since the equation (6.1) does not hold.

Example 2. Let $\mu$ be the Hamiltonian-minimal Lagrangian branched immersion with its right normal vector $j e^{\beta i}$ defined in the same way as Example 1. The function

$$
\alpha=\frac{2 A \mu_{0} \mu_{1}}{\left(\mu_{0}^{2}+\mu_{1}^{2}\right)^{2}}
$$




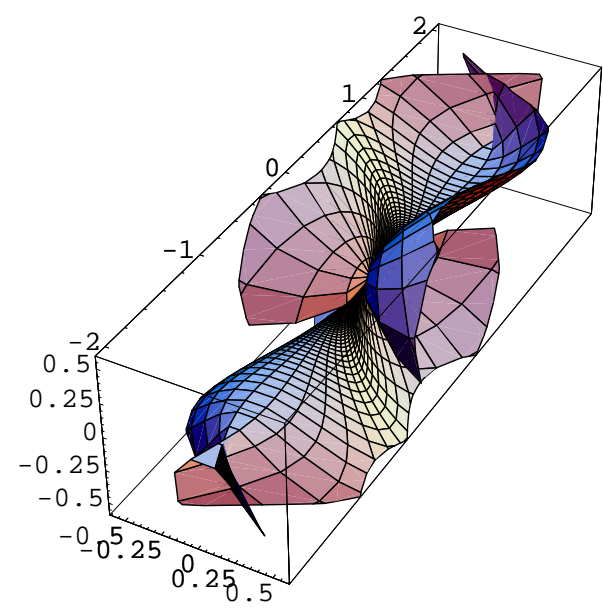

Figure 2. (Example 1) $U=\left\{x+y i \in \mathbb{C} \mid 0.5^{2} \leq x^{2}+y^{2} \leq 4^{2}\right\}, \mu_{0}=x, \mu_{1}=-y$, $A=1, \operatorname{Im} \lambda: U \rightarrow \operatorname{Im} \mathbb{H}$.

satisfies the equation $*(\mathrm{~d} \alpha)=(\mathrm{d} \beta)$. Then

$$
\nu=\alpha-j i e^{\beta i}=\frac{2 A \mu_{0} \mu_{1}}{\left(\mu_{0}^{2}+\mu_{1}^{2}\right)^{2}}-j i e^{\left\{A\left(\mu_{0}^{2}-\mu_{1}^{2}\right)\left(\mu_{0}^{2}+\mu_{1}^{2}\right)^{-2}\right\} i}
$$

is a Hamiltonian-minimal Lagrangian branched immersion with its right normal vector $j e^{\beta i}$. Indeed,

$$
\begin{aligned}
(\mathrm{d} \nu) & =(\mathrm{d} \alpha)+j e^{\beta i}(\mathrm{~d} \beta), \\
*(\mathrm{~d} \nu) & =(\mathrm{d} \beta)-j e^{\beta i}(\mathrm{~d} \alpha)=(\mathrm{d} \nu)\left(-j e^{\beta i}\right) .
\end{aligned}
$$

Since the left normal vector of $\nu$ is $-j e^{\beta i}$, we see that the Maslov form of $\nu$ is conformal by the equation (6.1). The image $\nu(M)$ is a part of a circular cylinder.

We define a smooth mapping $\lambda$ by $\underline{\hat{\nu}}=\underline{\hat{\mu}} \hat{\lambda}$. Then

$$
\begin{aligned}
& \lambda\left(\mu_{0}, \mu_{1}\right)=\frac{1}{\left(\mu_{0}^{2}+\mu_{1}^{2}\right)^{3}} \\
& \times\left[\left\{2 A \mu_{0}^{2} \mu_{1} e^{A \mu_{1}^{2}\left(\mu_{0}^{2}+\mu_{1}^{2}\right)^{-2} i}+\mu_{1}\left(\mu_{0}^{2}+\mu_{1}^{2}\right)^{2} i e^{A \mu_{1}^{2}\left(\mu_{0}^{2}+\mu_{1}^{2}\right)^{-2} i}\right\}\right. \\
& \left.+j\left\{-2 A \mu_{0} \mu_{1}^{2} e^{A \mu_{0}^{2}\left(\mu_{0}^{2}+\mu_{1}^{2}\right)^{-2} i}-\mu_{0}\left(\mu_{0}^{2}+\mu_{1}^{2}\right)^{2} i e^{A \mu_{0}^{2}\left(\mu_{0}^{2}+\mu_{1}^{2}\right)^{-2} i}\right\}\right]
\end{aligned}
$$

is a Lagrangian branched immersion with its right normal vector $j e^{\gamma i}$ which is not Hamiltonian-minimal by Theorem 4 . 


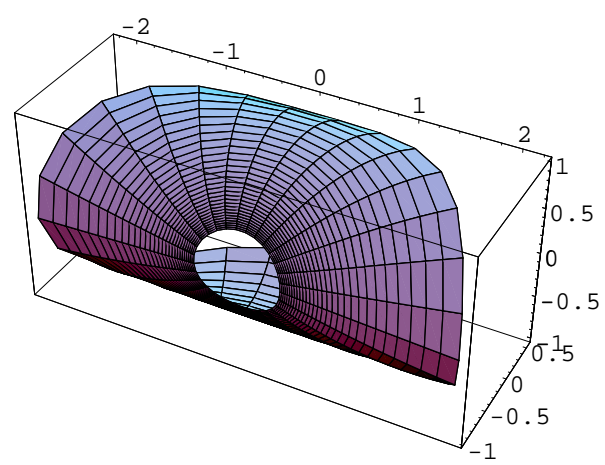

Figure 3. (Example 2) $U=\left\{x+y i \in \mathbb{C} \mid 0.68^{2} \leq x^{2}+y^{2} \leq 1.5^{2}\right\}, \mu_{0}=x, \mu_{1}=-y$, $A=1, \operatorname{Im}(i \nu): U \rightarrow \operatorname{Im} \mathbb{H}$.

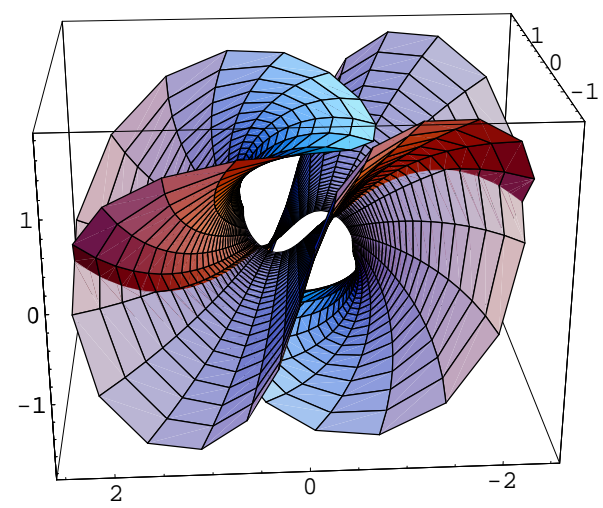

Figure 4. (Example 2) $U=\left\{x+y i \in \mathbb{C} \mid 0.68^{2} \leq x^{2}+y^{2} \leq 1.5^{2}\right\}, \mu_{0}=x, \mu_{1}=-y$, $A=1, \operatorname{Im}(i \lambda): U \rightarrow \operatorname{Im} \mathbb{H}$.

Let $x=\mu_{0}\left(\mu_{0}^{2}+\mu_{1}^{2}\right)^{-1}$ and $y=\mu_{1}\left(\mu_{0}^{2}+\mu_{1}^{2}\right)^{-1}$. Then

$$
\begin{aligned}
& \lambda=\left(2 A x^{2}+i\right) y e^{A y^{2} i}+j x\left(-2 A y^{2}-i\right) e^{A x^{2} i}, \\
& \lambda_{x}=4 A x y e^{A y^{2} i}+j\left(1+2 A x^{2} i\right)\left(-2 A y^{2}-i\right) e^{A x^{2} i}, \\
& \lambda_{y}=\left(2 A x^{2}+i\right)\left(1+2 A y^{2} i\right) e^{A y^{2} i}+j(-4 A x y) e^{A x^{2} i} .
\end{aligned}
$$

Hence the left normal vector of $\lambda$ is

$$
\begin{aligned}
& \lambda_{y} \lambda_{x}^{-1}=\frac{8 A x y\left(1+4 A^{2} x^{2} y^{2}\right)}{1+4 A^{2} x^{4}+16 A^{2} x^{2} y^{2}+4 A^{2} y^{4}+16 A^{4} x^{4} y^{4}} i \\
& +j\left\{\frac{1+16 A^{4} x^{4} y^{4}-4 A^{2}\left(x^{4}+y^{4}\right)}{1+4 A^{2} x^{4}+16 A^{2} x^{2} y^{2}+4 A^{2} y^{4}+16 A^{4} x^{4} y^{4}}\right.
\end{aligned}
$$




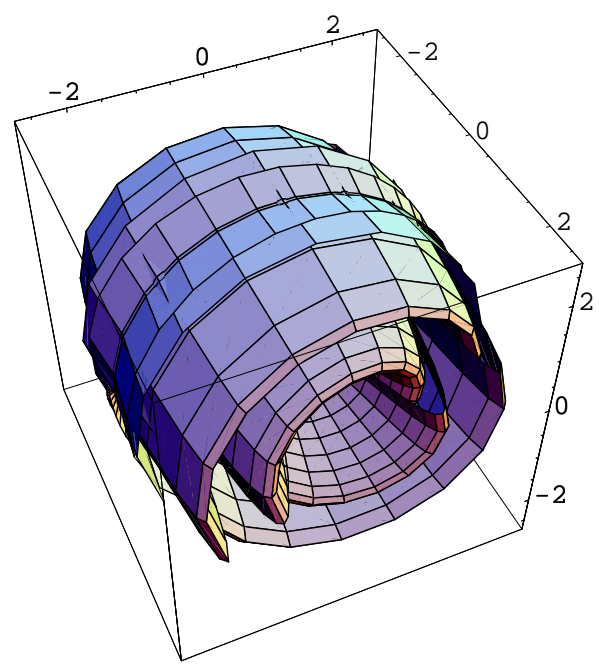

Figure 5. (Example 3) $U=\{x+y i \in \mathbb{C}|0.1 \leq| x|\leq 2.6| y \mid, \leq 2.6\}, \mu_{0}=x$, $\mu_{1}=-y, A=1, \operatorname{Im} \mu: U \rightarrow \operatorname{Im} \mathbb{H}$.

$$
\left.+\frac{4 A\left(x^{2}-y^{2}\right)\left(1+4 A^{2} x^{2} y^{2}\right)}{1+4 A^{2} x^{4}+16 A^{2} x^{2} y^{2}+4 A^{2} y^{4}+16 A^{4} x^{4} y^{4}} i\right\} e^{A\left(x^{2}-y^{2}\right)} .
$$

After long computation, we see that the Maslov form of $\lambda$ is not conformal by the equation (6.1).

Example 3. Let $\mu=\mu_{0} e^{(\beta-\gamma) i / 2}+j \mu_{1} e^{(\beta+\gamma) i / 2}$ with real-valued functions $\mu_{0}$ and $\mu_{1}$ on $M$ such that $\mu_{0}-\mu_{1} i$ is a non-constant complex holomorphic function vanishing nowhere on $M$. We assume that the mappings $\beta$ and $\gamma$ are given by the equations (5.8) and (5.9) with nonzero real number $A$ and $B=C=0$. Then

$$
\mu=\mu_{0} e^{A \mu_{1}^{2} i}+j \mu_{1} e^{A \mu_{0}^{2} i}
$$

is a Lagrangian branched immersion which is not Hamiltonian minimal with its right normal vector $j e^{\beta i}$ by Theorem 5 . We see that the Maslov form of $\mu$ is not conformal in a similar way as Example 1.

Let $p$ be a point in $M$ and

$$
\nu\left(\mu_{0}, \mu_{1}\right)=\int_{\mu_{0}(p)}^{\mu_{0}} e^{A t^{2} i} \mathrm{~d} t+j \int_{\mu_{1}(p)}^{\mu_{1}} e^{A t^{2} i} \mathrm{~d} t .
$$

Then $\nu$ is a Lagrangian branched immersion with its right normal vector $j e^{\beta i}$ which is not Hamiltonian-minimal. Indeed,

$$
\begin{aligned}
(\mathrm{d} \nu) & =e^{A \mu_{0}^{2} i}\left(\mathrm{~d} \mu_{0}\right)+j e^{A \mu_{1}^{2} i}\left(\mathrm{~d} \mu_{1}\right), \\
*(\mathrm{~d} \nu) & =e^{A \mu_{0}^{2} i}\left(\mathrm{~d} \mu_{1}\right)-j e^{A \mu_{1}^{2} i}\left(\mathrm{~d} \mu_{0}\right)=(\mathrm{d} \nu)\left(-j e^{A\left(\mu_{0}^{2}+\mu_{1}^{2}\right) i}\right) .
\end{aligned}
$$




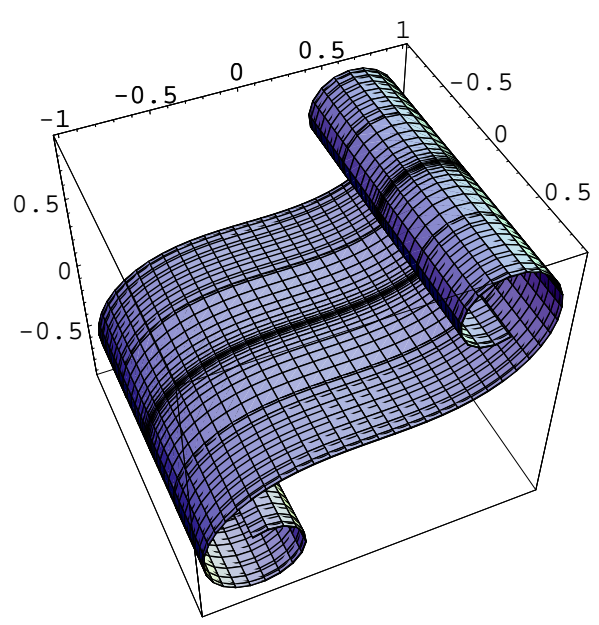

Figure 6. (Example 3) $U=\{x+y i \in \mathbb{C}|0.1 \leq| x|\leq 2.6| y \mid, \leq 2.6\}, \mu_{0}=x$, $\mu_{1}=-y, A=1, \operatorname{Im} \nu: U \rightarrow \operatorname{Im} \mathbb{H}$.

Since the left normal vector of $\nu$ is $-j e^{A\left(-\mu_{0}^{2}+\mu_{1}^{2}\right) i}$, we see that the Maslov form of $\nu$ is conformal by the equation (6.1). In the case where $M=\{x+y i \mid x, y \in \mathbb{R}\}=\mathbb{C}, A=1, \mu_{0}=x, \mu_{1}=-y$, and $p=0$, the map $\nu$ is a flat Lagrangian embedding given in [4].

Let us define a smooth mapping $\lambda$ by $\underline{\hat{\nu}}=\underline{\hat{\mu}} \hat{\lambda}$. Then

$$
\begin{aligned}
& \lambda\left(\mu_{0}, \mu_{1}\right)=\frac{1}{\mu_{0}^{2}+\mu_{1}^{2}} \\
& \times\left[\left\{\mu_{0} e^{-A \mu_{1}^{2} i} \int_{\mu_{0}(p)}^{\mu_{0}} e^{A t^{2} i} \mathrm{~d} t+\mu_{1} e^{A \mu_{0}^{2} i} \int_{\mu_{1}(p)}^{\mu_{1}} e^{-A t^{2} i} \mathrm{~d} t\right\}\right. \\
& \left.+j\left\{\mu_{0} e^{-A \mu_{1}^{2} i} \int_{\mu_{1}(p)}^{\mu_{1}} e^{A t^{2} i} \mathrm{~d} t-\mu_{1} e^{A \mu_{0}^{2} i} \int_{\mu_{0}(p)}^{\mu_{0}} e^{-A t^{2} i} \mathrm{~d} t\right\}\right] .
\end{aligned}
$$

is a Hamiltonian-minimal Lagrangian branched immersion with its right normal vector $j e^{\gamma i}$ by Theorem 5 . We should seek an alternative method to the equation (6.1) to conclude whether the Maslov form of $\lambda$ is conformal since the computation becomes very long.

\section{References}

1. Burstall, F. E., Ferus, D., Leschke, K., Pedit, F., and Pinkall, U.: Conformal geometry of surfaces in $S^{4}$ and quaternions. Lecture Notes in Mathematics 1772, Springer-Verlag, Berlin, 2002. 


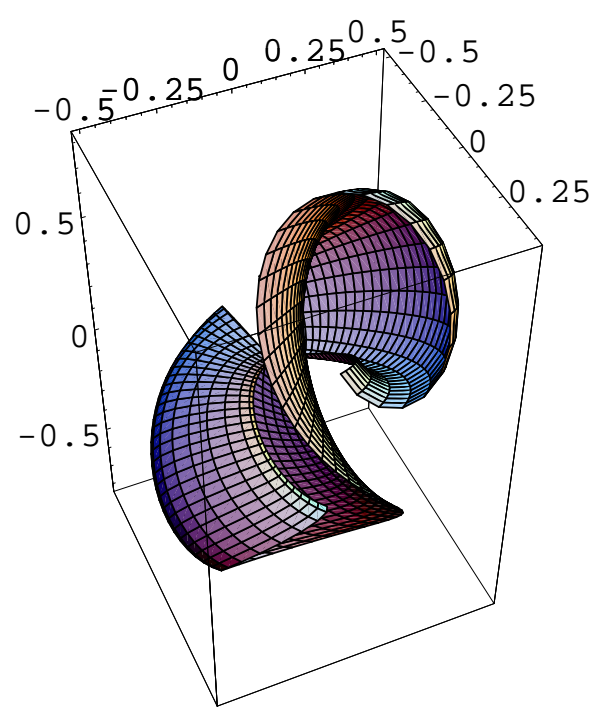

Figure 7. (Example 3) $U=\{x+y i \in \mathbb{C}|0.1 \leq| x|\leq 2.6| y \mid, \leq 2.6\}, \mu_{0}=x$, $\mu_{1}=-y, A=1, \operatorname{Im} \lambda: U \rightarrow \operatorname{Im} \mathbb{H}$.

2. Ferus, D., Leschke, K., Pedit, F., and Pinkall, U.: Quaternionic holomorphic geometry: Plücker formula, Dirac eigenvalue estimates and energy estimates of harmonic 2-tori. Invent. Math. 146 (3) (2001), 507-593.

3. Forster, O.: Lectures on Riemann surfaces, Graduate Texts in Mathematics 81, Springer-Verlag, New York, 1991.

4. Castro, I. and Urbano, F.: Lagrangian surfaces in the complex Euclidean plane with conformal Maslov form. Tohoku Math. J. (2) 45 (4) (1993), 565-582.

5. Hélein, F. and Romon, P.: Weierstrass representation of Lagrangian surfaces in four-dimensional space using spinors and quaternions. Comment. Math. Helv. 75 (4) (2000), 668-680.

6. Hélein, F. and Romon, P.: Hamiltonian stationary Lagrangian surfaces in $\mathbb{C}^{2}$. Comm. Anal. Geom. 10 (1) (2002), 79-126.

7. Oh, Y.-G.: Second variation and stabilities of minimal Lagrangian submanifolds in Kähler manifolds. Invent. Math. 101 (2) (1990), 501-519.

8. Pedit, F. and Pinkall, U.: Quaternionic analysis on Riemann surfaces and differential geometry. In: Proceedings of the International Congress of Mathematicians, Vol. II (Berlin, 1998). Doc. Math. Extra Vol. II (1998), 389-400.

9. Peters, G. P.: Soliton spheres. Dissertation, Technischen Universität Berlin, 2004.

10. Rodin, Y. L.: Generalized analytic functions on Riemann surfaces. Lecture Notes in Mathematics 1288, Springer-Verlag, Berlin, 1987.

11. Urakawa, H.: Calculus of variations and harmonic maps. Translations of Mathematical Monographs 132, Translated from the 1990 Japanese original by the author, American Mathematical Society, Providence, RI, 1993. 
12. Vekua, I. N.: Generalized analytic functions. Pergamon Press, London, 1962. 
moriya3.tex; $14 / 12 / 2007 ; 17: 11 ;$ p.26 